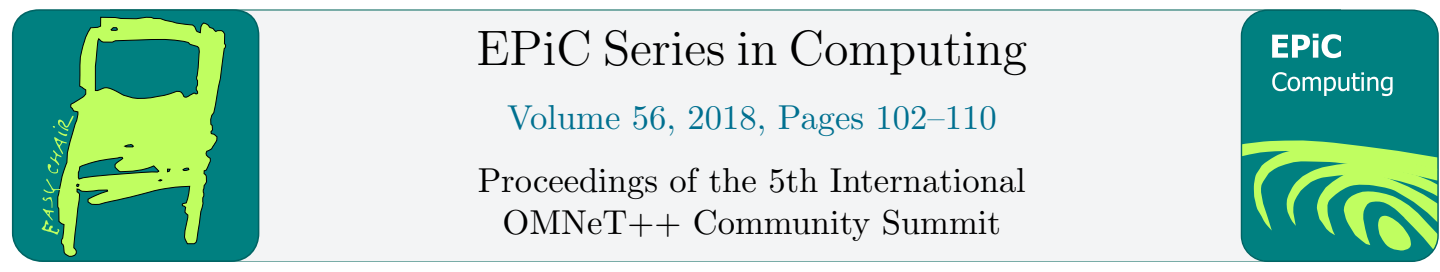

\title{
ResourSim - Evaluating the end-user Device usage
}

\author{
Jens Dede ${ }^{1}$ and Anna Förster ${ }^{1}$ \\ University of Bremen, Sustainable Communication Networks, Bremen, Germany \\ $\{j d$, afoerster\}@comnets.uni-bremen.de
}

\begin{abstract}
The optimization of apps on nowadays mobile phones, tablets and smart watches is mainly done with the focus on the energy constraints, i.e., the device battery lifetime. For some applications, this is adverse to their requirements and use cases like for background communication apps without direct user interaction. The framework ResourSim, as proposed in this work, aims on evaluating and optimizing the usage of device resources regarding the requirements of background tasks and the human user. This is achieved by replaying usage traces from real humans and adding arbitrary background job usage patterns. Furthermore, it is evaluated, if a statistical battery model is suitable for reflecting the real battery behaviour in ResourSim.
\end{abstract}

\section{Introduction}

Nowadays, virtually everybody owns at least one mobile device like a phone, tablet or even a smart watch and thus, carries a powerful computer most of the time. The operating systems on these devices - namely Android, iOS, watchOS, Wear OS etc. - try to optimize the battery lifetime using different technologies. Recent Android versions, for example, restrict background tasks to a minimum using a technology called Doze.

On the other hand, several applications and scenarios exist which require background activities without user interaction and therefore conflict with the energy saving features in nowadays devices. One class of these applications are opportunistic communication applications like the ODD model [2] or FireChat ${ }^{1}$. These kind of applications operate in the background using different technologies - mainly WiFi and Bluetooth - and create a kind of an ad-hoc network. These applications allow communication even if none of the infrastructure-based networks like WiFi or the cellular network are available for example due to a disaster, censorship or just missing coverage.

The idea of user-centered resource management [1] aims on optimizing the scheduling of background tasks according to the current user, or more exactly, the way a human uses the device. For that, the device usage is recorded using an Android app called ResourceMonitor ${ }^{2}$. This app collects data like the status of the screen, WiFi, cellular network connection, Bluetooth and the battery and allows to export this data for further offline analysis. It has been shown, that the usage of the devices highly depends on the human $[1,3]$ and therefore has to

\footnotetext{
${ }^{1}$ https: //www . opengarden.com/firechat.html

${ }^{2}$ https://github.com/ComNets-Bremen/ResourceMonitor
} 
performed individually for each user. Furthermore, the analysis for the scheduling has to be performed locally and offline per device as no Internet connectivity might be available due to the aforementioned reasons.

For the development of background scheduling algorithms based on and optimized for the user behaviour, an evaluation tool is required. ResourSim, as introduced in this work, is a simulation setup for OMNeT++ aiming on this gap. ResourSim replays usage traces from human users collected using ResourceMonitor and injects arbitrary background task patterns. It shows possible conflicts in the phone usage between the human and a background task. The objective is to optimize the activities of background tasks like opportunistic networks without any negative experience for the user.

This paper is structured as follows: Section 2 introduces ResourSim and the setup in OM$\mathrm{NeT}++$. Four example scenarios used for the evaluation of ResourSim are introduced in Section 3. These scenarios are evaluated and analyzed in Section 4. Section 5 gives some insights in the challenges of realistic battery modeling, whereas Section 6 concludes this work.

\section{ResourSim}

ResourSim is a simulation setup for the $\mathrm{OMNeT}++$ simulation environment. In contrast to the majority of simulations run in $\mathrm{OMNeT}++$, ResourSim does not simulate a communication network, but uses the discrete event simulation capabilities of $\mathrm{OMNeT}++$ to run the eventbased traces collected using ResourceMonitor. The ResourSim setup including the example traces used in this work is available on GitHub ${ }^{3}$.

Figure 1 shows the modules currently implemented in ResourSim. Three types of modules are available:

Injectors inject events into the network. Currently, the userEventInjector and the backgroundServiceInjector are available.

Manager forwards the events from the injectors to the sink modules.

Sinks are all other modules. They reflect the status of the entity and collect statistics about the states and collisions between different events.

The userEventInjector module reads the real user traces collected using ResourceMonitor and creates the corresponding events. For that, the traces are imported as an XML file which contains the events in the following format:

<event data_type="WiFiStatus" time="2017-12-02T18:30:09Z" timestamp_s

$\hookrightarrow=" 3312653.0 "$ wifi_status $=" 4 ">3312653.0</$ event $>$

Besides the type of the event (WiFiStatus) and the time in different representations, wifi_status="4" indicates that WiFi is connected. Similar events exist for Bluetooth, screen status, cellular network, battery, flight mode and the traffic statistics. For each event in the $\mathrm{XML}$ file, an event in OMNeT++ represented by a message containing the additional information is created.

The event messages created by the backgroundServiceInjector module are similar to the user messages. They are created according to the parameters defined in the omnetpp.ini:

start The time when the event ist injected the first time.

\footnotetext{
${ }^{3}$ https://github.com/ComNets-Bremen/ResourSim
} 


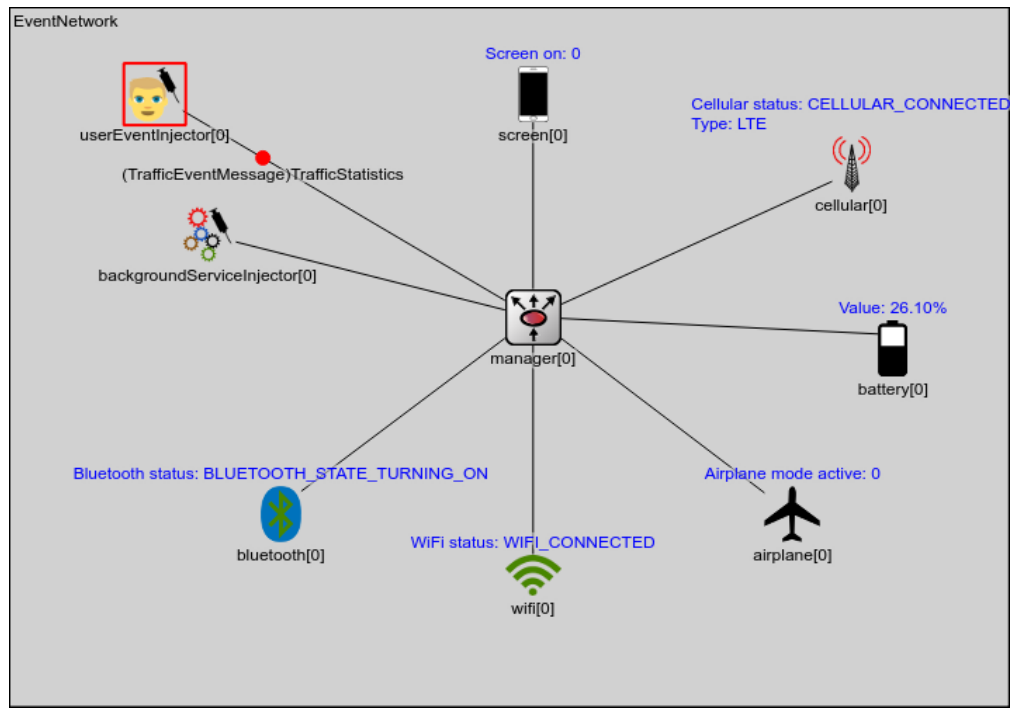

Figure 1: The Configuration of ResourSim

stop The last time the event is injected.

duration How long does an event occupy the resource?

type The type of the event (e.g. WiFi).

repetition The time between two consecutive events, inter-event time.

These messages are sent to the central manager module which forwards the events to the sink modules. Each sink module represents one entity of a real device:

- The screen module reflects the current status of the screen. It is assumed that the user is actively using the device if the screen is on.

- The connection status and the connection type of the cellular network interface is reflected by the cellular module.

- Energy-related data like the battery status (charging, discharging) and the battery level are modeled by the battery model.

- When the user switches the device to flight mode, it will be mentioned by the airplane model.

- The wifi module reflects the current status of the WiFi connection.

- The bluetooth module reflects the current status of the Bluetooth interface.

Each sink module records its current state for later statistical analysis. Furthermore, the WiFi, Bluetooth and screen modules track the current user (i.e. background or human) and record collisions between both. For that, it is assumed that WiFi and Bluetooth can only be used either by the human or by the background task. The screen status represents if the user 
is actively using the device. In this case, a background tasks should not perform any heavy background operation like data processing. Additionally, it is assumed that once a task has been started, it cannot be interrupted by another tasks. If this happens, a collision will occur. There are two types of collisions:

User Collision The user requests a resource. If a resource like WiFi for Internet connectivity is not available at this time, this will result into a negative user experience and thus has to be avoided.

Background Collision The background service requests a resource (CPU time, WiFi etc.) which is not available, i.e., occupied by the human user. The drawback for the user in this case is mostly negligible. The drawback for the background process depends on the type of the service.

The number of collisions is counted and normalized to collisions per day. These values are used to estimate the user experience for different scenarios in Section 4. The battery sink plays a special role and will be discussed later in Section 5. The following Section 3 describes the reference scenarios used in this work.

\section{Evaluation Scenario Description}

To evaluate and demonstrate the functionality of ResourSim, four reference scenarios for assumed background tasks are defined in the omnetpp.ini. For each them, the start and end time, duration, service type and repetition (inter service time) can be defined. The focus is on the latter three as the start time is set to one and the stop time is not used for all scenarios.

For a better understanding, the focus is on the WiFi and CPU or screen usage: To determine if the human is currently using the device, the screen status is used. This corresponds to CPU usage for the background task, i.e. if the screen is on, no heavy CPU job should be started by the background task and vice versa. WiFi is assumed to be only accessible either by the user or by the background task. This is valid as both are assumed to have contradictory requirements: The user needs Internet connectivity whereas the background task requires network scanning and direct data transfer to other nodes which is not possible at the same time. The following scenarios are defined:

Scenario I does not assume any background service at all. Only the human usage traces are replayed. This scenario will be used for testing the battery module in Section 5 .

Scenario II assumes regular background scanning for WiFi nodes in proximity which reflects a neighbor discovery. The service type is set to $\mathrm{WiFi}$, the inter service time is set to uniform(500s, 700s) which is a uniform distribution between 500 and 700 seconds. For the duration of each scan, a truncated non-negative normal distribution with $\mu=2 \mathrm{~s}$ and $\sigma=1 \mathrm{~s}$ is used and defined by truncnormal (2s, $1 \mathrm{~s})$.

Scenario III extends the Scenario II with CPU usage. It is assumed that the data collected during the WiFi scans is processed once per hour. For that, some CPU time is required. This is done by adding a second background task of type CPU. The time between two executions is assumed to be normal distributed with $\mu=3600 \mathrm{~s}$ and $\sigma=10 \mathrm{~s}$. The duration is again defined as a truncated non-negative normal distribution with $\mu=10 \mathrm{~s}$ and $\sigma=5 \mathrm{~s}$. The corresponding lines in the omnetpp. ini are normal (3600s, 10s) and truncnormal (10s, 5s), respectively. 


\begin{tabular}{|r|r|c|c|}
\hline & Collision Type & User 1 $\left[\frac{1}{\text { day }}\right]$ & User 2 $\left[\frac{1}{\text { day }}\right]$ \\
\hline \multirow{4}{*}{ Scenario II } & WiFi user & 0.125 & 0.069 \\
& WiFi background & 110.366 & 88.090 \\
& usage user & 0.0 & 0.0 \\
& usage background & 0.0 & 0.0 \\
\hline \multirow{4}{*}{ Scenario III } & WiFi user & 0.062 & 0.094 \\
& WiFi background & 110.420 & 88.199 \\
& usage user & 0.187 & 0.484 \\
& usage background & 1.572 & 5.163 \\
\hline \multirow{3}{*}{ Scenario IV } & WiFi user & 4.179 & 2.682 \\
& WiFi background & 536.560 & 437.113 \\
& usage user & 0.241 & 0.369 \\
& usage background & 1.712 & 5.104 \\
\hline
\end{tabular}

Table 1: Average collisions per day by collision type for two user traces.

User collision: user is negatively affected, background collision: background task is negatively affected.

Scenario IV assumes an opportunistic communication application heavily using WiFi and requesting CPU time. The CPU usage is the same as defined in Scenario III, the WiFi inter service time is set to a fixed values of $120 \mathrm{~s}$. The duration is set to a non-negative truncated normal distribution with $\mu=10 \mathrm{~s}$ and $\sigma=5 \mathrm{~s}$ (truncnormal(10s, 5s)).

For the evaluation of these scenarios, two traces of mobile phones of two different users are used. These traces were selected to reflect significantly different user behaviours. The trace of user 1 contains data of 3084 hours (129 days), the trace of user 2 is longer and contains data for 7670 hours (320 days).

These scenarios will be evaluated with the user traces in the following Section 4 .

\section{Example Scenario Analysis}

In this section, the traces of the two users are run for the scenarios defined in Section 3. The number of collisions per day for the different tasks and users are shown in Table 1. Scenario I is not mentioned as it is a replay scenario without any injected background task and therefore, no collisions occur.

It can be seen that a moderate WiFi usage as defined in Scenario 2 barely affects the human user but with approximately 100 conflicts per day for both user traces has a notable influence on the background task. Similar can be observed for the Scenario III. In Scenario I and II, both users will in average less than once per day notice drawbacks in the daily usage of their devices. Only in case of heavy usage of the device by the background task as defined in the Scenario IV, the user will face a notable decreased experience: User 1 will in average collide four times per day in the WiFi usage with the background task. The same happens for the second user in average 2.7 times per day which can (depending on the user expectation) be considered as a major effect on the daily usage.

In contrast to the user, the background task have to deal with a significantly higher amount of collisions. This is obvious as the duration of the events injected by the background task are short (couple of seconds) whereas a user normally uses the device resources for a longer period of time. Whether the background task can handle these collisions depends on the used 
technology and protocols and is out of scope of this work.

Another aspect which has not been discussed in this section is the affect on the energy consumption of the background task. The challenges for this will be discussed in Section 5 .

\section{$5 \quad$ Battery and Energy Consumption Modeling}

In the previous section, the collisions in the usage of mobile device resources between a human user and a background task have been analyzed. However, the influence on the device battery has been neglected. Depending on the device resources occupied by the background task, the influence on the battery lifetime can be remarkable. In a worst case, the user might have a phone with a battery completely drained by a background task without the chance of charging it where he is used to have a almost fully charged device.

The (dis)charging of a phone depends on a variety of different parameters like (but not limited to) the phone hardware and the battery capacity, version of the operating system, installed and used apps, age of the battery, the current user activity etc. For example in the INET framework ${ }^{4}$, modules for the energy generation, storage and consumption exist. Due to the large variety of different systems and parameters, the setup of such a model for ResourSim would be a challenging task. Alternatively, one could create a power model by analyzing the traces by the battery level and the used resources as done in [3] for the Android G1 in 2009. This is possible, but complex as it has to be done in theory every time the user (un)installs or updates an application as it might significantly influence the energy consumption. In this section, it is evaluated how close one can get to reality with a statistical battery model.

The first approach is to solely use the available battery data from the ResourceMonitor trace. This fails in case of injected background tasks which require energy as the battery get discharged faster. While charging again, the model also have to consider the new battery level and adapt accordingly. Here, an extrapolation of the battery values is required.

In this work, the existing traces are used to evaluate, if a simplified statistical model can be used. For this, the replay Scenario I is used. There, no additional background services are setup and the model can easily be compared to the real values. In case of a perfect matching model, the difference between the real and the calculated values should be zero.

The charging and discharging processes are evaluated separately for each user. The change in the values is normalized to percent points per hour. The following methods are evaluated:

1. The overall mean charging and mean discharging rate per hour, calculated for the complete user trace.

2. The overall median charging and median discharging rate per hour, calculated for the complete user trace.

3. An array of the mean charging and mean discharging values in dependence of the current battery level.

4. An array of the median charging and median discharging values in dependence of the current battery level.

All these values can easily be created from the existing traces from ResourceMonitor and setup in the corresponding sections in the omnetpp.ini. Table 2 shows the input values for the simulation.

\footnotetext{
${ }^{4}$ https://inet.omnetpp.org
} 


\begin{tabular}{|c|r|c|c|}
\hline & & User 1 $\left[\frac{\%}{\text { hour }}\right]$ & User 2 [\% $\left.\frac{\%}{\text { hour }}\right]$ \\
\hline \multirow{2}{*}{ Mean Values } & Charging & 47.85 & 58.59 \\
& Discharging & -23.95 & -17.78 \\
\hline \multirow{2}{*}{ Median Values } & Charging & 37.50 & 56.25 \\
& Discharging & -8.2 & -7.96 \\
\hline
\end{tabular}

Table 2: The mean and median values for charging and discharging the device battery in percent points per hour for user 1 and user 2 .

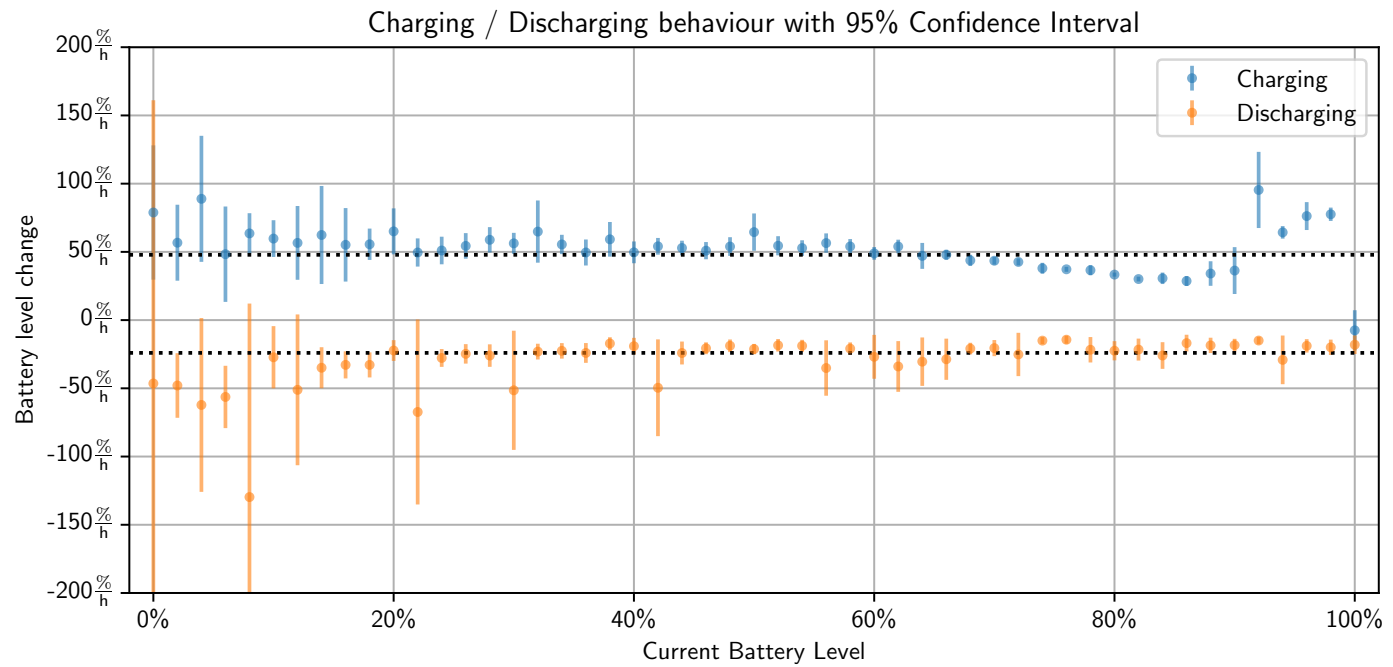

Figure 2: The mean relative change of the battery level in percent points per hour in dependency of the current battery level for user 1 with $95 \%$ confidence interval. The dotted lines represent the mean charging and mean discharging values, respectively.

The array values are in the format <current value>: <change per hour>, and defined as follows in the omnetpp.ini:

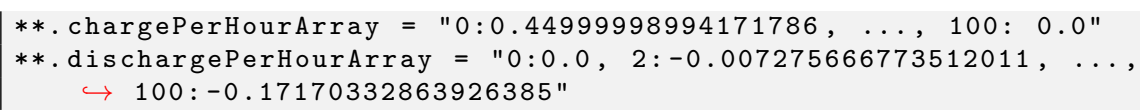

An example plot of this array showing the mean charging and mean discharging per hour for user 1 is shown in Figure 2. One can see that for example the discharging at a battery level of $12 \%$ is in average $-50 \frac{\%}{\mathrm{~h}}$. The increase in the confidence interval for lower battery levels corresponds to the charging behaviour of the user: user 1 keeps the battery level high, only few measurements for low battery levels, i.e. less than $20 \%$ are available. The dotted lines represent the means for the charging and discharging values.

The four methods are applied to the two user traces and the calculated battery level and the measured real battery level are compared. The results are shown in Table 3. One can see that for all four methods, the differences between the real and the calculated battery level are $42 \%$ and $57 \%$ with a notable standard deviation and are therefore not suitable for an easy to 


\begin{tabular}{|l|c|c|}
\hline \multirow{2}{*}{ Setup } & \multicolumn{2}{|c|}{ Delta to real value } \\
\cline { 2 - 3 } & Mean $(\mu)$ & Standard deviation $(\sigma)$ \\
\hline User 1, mean values & $56.97 \%$ & $22.77 \%$ \\
User 1, median values & $44.55 \%$ & $20.17 \%$ \\
User 1, array of mean values & $56.06 \%$ & $20.97 \%$ \\
User 1, array of median values & $43.92 \%$ & $20.00 \%$ \\
\hline User 2, mean values & $42.06 \%$ & $26.54 \%$ \\
User 2, median values & $45.76 \%$ & $27.50 \%$ \\
User 2, array of mean values & $42.02 \%$ & $26.80 \%$ \\
User 2, array of median values & $45.31 \%$ & $27.64 \%$ \\
\hline
\end{tabular}

Table 3: The mean values and the standard deviation of the difference between the real and the estimated battery levels using different statistical methods.

use and implement statistical battery model. It is part of the future work to further investigate this challenge and implement better models for the charging and discharging behaviour.

\section{Conclusion}

In this work, ResourSim has been introduced. It is a simulation setup for OMNeT++ which helps to easily analyze the effect of background tasks on the daily usage on real user devices like smartphones and tablets. The real user behaviour is taken from traces collected using the ResourceMonitor app. Arbitrary background tasks like CPU or WiFi usage can be injected and the conflicts between the human user and the background task can be analyzed. This helps to increase the understanding in the interdependency of both and optimize the scheduling of background activities using new scheduling technologies on mobile devices. ResourSim has been evaluated using different example scenarios for two real user traces.

Furthermore, it has been evaluated whether statistical methods can be used to model the battery behaviour and if this is suitable to match the requirements for ResourSim. It has been shown, that the charging and discharging behaviour of nowadays smartphones is too dynamic to be reflected by simplistic statistical methods.

For the future work, the authors are going to evaluate other battery charging and discharging models and implement them in ResourSim as the battery level is one of the most crucial properties for a user on a mobile device. In addition to that, more complex evaluation scenarios are going to be implemented. One aim is a fine-grained analysis of the usage multiple resources for both, the human user and the background task.

Finally, the authors would like to invite the interested reader to contribute to the ResourSim project and comment on the implemented methods and technologies.

\section{References}

[1] Jens Dede, Asanga Udugama, and Anna Förster. Poster: User-centered resource management for background services. http://dx.doi.org/10.13140/RG.2.2.16922.13769, 2017.

[2] Anna Foerster, Asanga Udugama, Carmelita Görg, Koojana Kuladinithi, Andreas Timm-Giel, and Alejandro Cama-Pinto. A Novel Data Dissemination Model for Organic Data Flows, pages 239-252. Springer International Publishing, Cham, 2015. 
[3] Alex Shye, Benjamin Scholbrock, and Gokhan Memik. Into the wild: Studying real user activity patterns to guide power optimizations for mobile architectures. In Proceedings of the 42Nd Annual IEEE/ACM International Symposium on Microarchitecture, MICRO 42, pages 168-178, New York, NY, USA, 2009. ACM. 